\title{
Fatores relacionados ao prognóstico de vítimas de traumatismo cranioencefálico: uma revisão bibliográfica
}

\author{
Aline Silva Jerônimo ${ }^{1}$, Sheila Cristiane Evangelista Creôncio², Denise Cavalcanti \\ José Carlos de Moura ${ }^{4}$, Ricardo Argenton Ramos ${ }^{4}$, Alcieros Martins da Paz ${ }^{5}$ \\ Universidade Federal do Vale do Francisco, Petrolina, PE, Brasil.
}

\section{RESUMO}

O traumatismo cranioencefálico (TCE) é uma agressão traumática ao cérebro, em consequência de um trauma externo, resultando em alterações cerebrais momentâneas ou permanentes e comprometimento das habilidades cognitivas ou físicas. O presente artigo tem como objetivo apresentar uma revisão bibliográfica sobre os fatores relacionados ao prognóstico de vítimas de TCE. O estudo compreende um levantamento bibliográfico para o conhecimento e melhor abordagem sobre o tema, possibilitando uma reflexão sobre esses fatores. Com base na literatura e análise dos estudos, evidenciou-se que a baixa pontuação na Escala de Coma de Glasgow tem sido um forte indicador de mau prognóstico das vítimas de TCE. Além disso, a influência de fatores como gravidade das lesões, tipo de lesão intracraniana, idade, hipóxia e hipotensão arterial também se apresentou como determinante sobre a evolução dos pacientes e esteve relacionada ao prognóstico de vítimas de TCE. Podemos observar que nos últimos anos se têm aprofundado os estudos no intuito de se buscar os fatores prognósticos para o TCE, e a identificação desses indicadores tem representado um grande avanço na busca de alternativas para orientar o tratamento do paciente e se estimar o resultado final.

\section{PALAVRAS-CHAVE}

Traumatismos craniocerebrais, prognóstico, morbidade.

\begin{abstract}
Factors associated with prognosis of traumatic brain injury: a literature review

Traumatic brain injury is an injury to the brain trauma as a result of external trauma, resulting in momentary or permanent brain changes and impairment of cognitive abilities or physical functioning. This article aims at reviewing the literature on the factors related to the prognosis of traumatic brain injury. The study comprises a literature to the best knowledge and approach to the subject, providing a reflection on these factors. Based on the literature and analysis of the studies showed that the low score on the Glasgow Coma Scale has been a strong indicator of poor prognosis of traumatic brain injury. Furthermore, the influence of factors such as brain injury, severity of injury, type of intracranial injury, age, hypoxia and hypotension were also presented as determinants of the evolution of patients and were related to the prognosis of traumatic brain injury. We observed that in recent years has deepened the studies in order to seek the prognostic factors for traumatic brain injury, where the identification of these indicators has represented a major breakthrough in the search for alternatives to guide patient treatment and to estimate the end result.
\end{abstract}

\section{KEYWORDS}

Craniocerebral trauma, prognosis, morbidity.

1 Enfermeira, especialista em Gestão em Saúde, mestranda em Ciências da Saúde e Biológicas pela Universidade Federal do Vale do São Francisco (Univasf), Petrolina, PE, Brasil.

2 Enfermeira especialista em Urgência, mestranda em Ciências da Saúde e Biológicas pela Univasf, Petrolina, PE, Brasil.

3 Terapeuta ocupacional, mestranda em Ciências da Saúde e Biológicas pela Univasf, Petrolina, PE, Brasil.

4 Professor adjunto da Univasf, Petrolina, PE, Brasil.

5 Cirurgiã-dentista, mestre em Saúde Coletiva pela Universidade Federal de Pernambuco (UFPE), Recife, PE, Brasil. 


\section{Introdução}

A elevação gradual dos índices de mortalidade por causa de acidentes e violência tornou-se um grave e sério problema de saúde da população dos países industrializados. O aumento progressivo de vítimas de traumas mecânicos vem determinando o crescimento dessas mortes. Os traumas mecânicos atualmente vêm sendo classificados como a principal causa de óbito e sequelas na população abaixo de 45 anos de idade. ${ }^{1}$

Entre os traumas mecânicos, o traumatismo cranioencefálico (TCE) é o principal determinante de óbitos e sequelas em politraumatizados. ${ }^{2}$ Traumatismo cranioencefálico é qualquer agressão traumática ao cérebro que tenha como consequência lesão anatômica como fratura de crânio ou lesão do couro cabeludo, comprometimento funcional das meninges, encéfalo e seus vasos ou alterações cerebrais momentâneas ou permanentes, de natureza cognitiva ou de funcionamento físico. ${ }^{3}$

Mais de 1 milhão de pessoas ficaram inválidas em decorrência de traumas mecânicos no Brasil nos últimos 10 anos, sendo os principais responsáveis por essas taxas os acidentes de trânsito. Dependendo da instituição analisada, o internamento por trauma mecânico pode chegar a atingir índices acima de $40 \%$, sendo, por isso, considerado um problema de saúde pública. ${ }^{2-4}$

No que diz respeito principalmente à sua gravidade, o TCE pode ocasionar incapacidade ou óbito, mudar permanentemente as habilidades e suas perspectivas e modificar a vida de seus familiares, além de deixar sequelas, despesas indiretas, perdas de anos de vida e de produtividade, reabilitação, custos das perdas materiais, entre outros. Entre as principais causas de TCE, podemse citar acidentes automobilísticos, atropelamentos, acidentes ciclísticos e motociclísticos, agressões físicas, quedas, lesões por arma de fogo, entre outras menos frequentes. ${ }^{5}$

A mortalidade dos pacientes vítimas de TCE é bem expressiva, representando um percentual em torno de $40 \%$; e esse elevado índice não se limita apenas aos países desenvolvidos, estando presente em todo o mundo. No Brasil, em 2008, as regiões Sudeste e Nordeste tiveram os maiores índices de mortalidade por essas causas. ${ }^{4}$

O sexo mais frequentemente acometido pelo TCE é o masculino, o que pode ser justificado pelas diferenças nas situações de riscos aos quais ambos estão envolvidos. Um estudo realizado por Moura et al., ${ }^{2}$ no ano de 2011, que compara homens com mulheres, refere uma proporção de 6,21:1. Relativamente à alta mortalidade ocasionada pelo TCE, pode-se verificar um número maior de mortos entre os sujeitos mais velhos. No público infantil, verifica-se como a principal causa de morte o TCE, no entanto crianças apresentam mortalidade menor do que adultos. ${ }^{6}$
É indispensável destacar que nos últimos anos o Brasil vem apresentando um crescimento exacerbado na frota de motocicletas, o que vem resultando em aumento crescente no número de acidentes envolvendo esse tipo de veículo. Em determinadas regiões do país, esse fator chega a ser a principal etiologia desse evento estudado. ${ }^{2}$

\section{Revisão de literatura}

Nos últimos anos temos visto um crescente avanço nos estudos relacionados às causas e consequências dos traumatismos cranioencefálicos. Diversificações para categorizar a gravidade de um TCE podem ser evidenciadas com frequência na literatura. Apesar de vários estudos publicados acerca do tema, ainda são insuficientes os estudos relacionados ao prognóstico dessas vítimas. ${ }^{7-8}$

$\mathrm{Na}$ literatura, vários fatores são relacionados a pior prognóstico em pacientes vítimas de TCE, e os mais citados são: pontuação igual ou menor que 8 na Escala de Coma de Glasgow (ECG) na admissão; idade acima dos 60 anos; alterações tomográficas evidenciando lesão axonal difusa ou edema cerebral; pupilas com reflexos fotomotor abolidos; hipotensão arterial na admissão; hipertermia e sexo masculino. ${ }^{1}$

Muñoz-Céspedes et al., em estudo sobre os fatores relacionados com o prognóstico de vítimas de TCE, afirmam que a determinação de um prognóstico após um TCE constitui uma questão importante do ponto de vista tanto clínico quanto do paciente e da família.

A busca de indicadores relacionados ao prognóstico de vítimas de TCE é dificultada por causa do grande número de variáveis que influenciam a recuperação pós-trauma desses pacientes. ${ }^{8}$ Fatores como consequências pós-trauma, índices de gravidade, indicadores neurológicos, fatores pré-mórbidos, observações clínicas, medidas fisiológicas e dados radiológicos e laboratoriais são elementos de investigação para se estabelecerem indicadores seguros que permitam chegar ao prognóstico em médio e longo prazo de uma vítima de trauma. ${ }^{7-8}$

Para Oliveira et al., ${ }^{9}$ a predição do prognóstico é um dos principais problemas associados ao TCE grave. Cambier et al. ${ }^{10}$ afirmam que o prognóstico do paciente vítima de TCE depende dos aspectos anatomoclínicos e evolutivos do trauma, dentre os quais podemos destacar as concussões cerebrais, as contusões cerebrais e os hematomas intracranianos, subdivididos em hematoma extradural, hematoma subdural e hematoma intracerebral. Esses autores afirmam, ainda, que as sequelas se apresentam com mais frequência após traumatismos graves, definidos por um escore inferior a 8 na Escala de Coma de Glasgow, amnésia pós-traumática de mais de 24 horas, déficits neurológicos, alterações psíquicas, 
epilepsia pós-traumática, alterações vasculares, cefaleias, vertigens e distúrbios do sono.

Muñoz-Céspedes et al. ${ }^{7}$ também citam que a natureza, a intensidade, a duração do tratamento e o manejo desses pacientes são condicionados em grande parte pelo prognóstico dessas vítimas. Esses estudos afirmam, ainda, que um dos principais fatores que determinam a evolução desses pacientes são as alterações neuropsicológicas pós-traumáticas, pois condicionam tanto o grau de independência funcional como o estabelecimento de relações familiares e sociais satisfatórias. ${ }^{7}$

Também em relação aos fatores relacionados com a mortalidade de crianças com TCE grave, a literatura evidencia a relação de alguns fatores com maior mortalidade, tais como hipertensão intracraniana, hipotensão arterial, hipóxia, hipercapnia, lesões intracranianas e traumatismos associados ao TCE, servindo como base para a predição do prognóstico dessas vítimas. ${ }^{11} \mathrm{~A}$ hipertensão intracraniana, a hiperglicemia, os distúrbios de coagulação sanguínea e algumas lesões intracranianas também estiveram relacionados com um pior prognóstico de crianças com TCE grave. ${ }^{11}$

\section{Métodos}

Para alcançar os objetivos propostos neste estudo, realizou-se levantamento bibliográfico on-line nas bases de dados da Biblioteca Virtual de Saúde (BVS), Literatura Latino-Americana e do Caribe em Ciências da Saúde (Lilacs) e Scientific Electronic Library Online (SciELO).

Foram utilizados, para busca dos artigos, os seguintes descritores e suas combinações nas línguas portuguesa e inglesa: "trauma craniano", "prognóstico" e "morbidade".

Os critérios de inclusão definidos para a seleção dos artigos foram: artigos publicados em português, artigos na íntegra que retratassem a temática referente ao tema abordado e que a metodologia consentisse obter evidências sobre a associação dos descritores utilizados, independentemente do método de pesquisa utilizado, e artigos publicados e indexados nos referidos bancos de dados nos últimos dez anos, ou seja, entre os anos de 2002 e 2012. Artigos de anos anteriores foram citados na medida de sua importância para o tema em questão.

Em seguida, foram selecionadas, criteriosamente, todas as bibliografias consideradas relevantes e pertinentes ao objetivo da pesquisa, que, juntas, somaram informações necessárias para o desenvolvimento do tema proposto neste estudo.

A análise dos estudos selecionados, em relação ao delineamento de pesquisa, possibilitou observar, des- crever e classificar os dados, com o intuito de reunir o conhecimento produzido sobre o tema explorado na revisão. Após leitura atenta e minuciosa de cada material, as informações colhidas foram analisadas e discutidas no trabalho.

\section{Resultados}

Após análise e revisão da literatura sobre os fatores que influenciaram a evolução de pacientes com TCE, foi demonstrado nos estudos abordados que a gravidade clínico-neurológica inicial, medida por meio da ECG, tem a maior influência significativa na evolução dos pacientes, evidenciando que a manifestação clínica inicial aponta a gravidade das lesões primárias e secundárias associadas ao TCE. ${ }^{4}$

Assim como a ECG, diversos outros fatores como a suscetibilidade do cérebro à lesão, extensão e gravidade das lesões, presença de lesões globais ou focais, lesões associadas e resposta inicial ao tratamento também foram citados como úteis para determinar a evolução dos casos de vítimas de TCE. ${ }^{3,7-9,11-14}$

As sequelas neurológicas graves nos pacientes que se recuperam de um TCE também podem determinar uma qualidade de vida muito prejudicada. Em estudo de Dantas Filho et al., ${ }^{12}$ a presença de hipóxia nos pacientes na sua admissão também exerceu influência bastante significativa, pois foi indicativa de mau prognóstico, visto que $40 \%$ desses pacientes evoluíram a óbito. Os episódios de hipóxia e hipotensão, quando verificados na admissão hospitalar, também foram fatores que se inseriram na tentativa de determinar mais precisamente o prognóstico de pacientes vítimas de TCE. ${ }^{1,9,12}$

Os fatores relacionados à idade também foram um tema abordado na literatura com frequência. Apesar de a idade avançada ser descrita como variável independente associada a um pior prognóstico e da tradicional expectativa de melhor evolução entre os mais jovens e de pior evolução entre os mais velhos, estudos ainda são discordantes em relação à influência desse fator no prognóstico de pacientes vítimas de TCE. ${ }^{1,4,7,8,12}$

Por outro lado, as observações de Melo et al. ${ }^{1}$ corroboram as de Dantas Filho et al. ${ }^{12}$ quando estes últimos relatam em seu estudo o fato de pacientes com maior faixa etária não sobreviverem tempo suficiente para receber atendimento médico, sendo atendidos apenas os pacientes com TCE sobreviventes.

A presença de febre durante a internação hospitalar também foi citada como relacionada a pior prognóstico. ${ }^{1}$ Nesses casos, a febre apresentou significado maior como fator preditivo de morbidade e letalidade entre os pacientes. ${ }^{1}$ 
Outros fatores também foram citados nos estudos analisados como relacionados a pior prognóstico nas vítimas de TCE e a maiores taxas de letalidade. No estudo de Melo et al. ${ }^{1}$ e Carvalho et al., ${ }^{11}$ achados tomográficos evidenciando lesão axonal difusa ou edema cerebral, pupilas sem reflexo fotomotor, pacientes do sexo masculino e acidentes que envolvem meios de transporte foram fatores que puderam auxiliar na compreensão de fatores determinantes para o prognóstico dos pacientes vítimas de TCE.

Outros estudos também citam fatores como comorbidades médicas prévias e o tempo das intervenções clínicas e cirúrgicas como relacionados à determinação e à evolução dos casos de TCE. ${ }^{7,13,14}$

\section{Discussão}

Vários estudos têm demonstrado que a gravidade do TCE de acordo com a ECG tem sido usada como um dos mais importantes preditores no desfecho dessas vítimas, e a baixa pontuação na ECG foi o principal fator relacionado com pior prognóstico dos pacientes vítimas de TCE. ${ }^{1,4,7,9,12}$

No estudo de Melo et al., ${ }^{1}$ realizado por meio da revisão de prontuários médicos de vítimas de TCE, foi verificado que pontuação $\leq 8$ na ECG na admissão hospitalar exerceu influência negativa no prognóstico desses pacientes. Em uma análise com 63 vítimas de TCE em seguimento ambulatorial entre seis meses e três anos após o evento traumático, vários estudos têm demonstrado que grupos de vítimas com pontuação 8 na ECG apresentam piores consequências pós-traumáticas quando comparados aos demais. ${ }^{8}$

Esses resultados confirmam as afirmações de três outros autores. Em estudo de Sousa ${ }^{8}$ e Muñoz-Céspedes et al., ${ }^{7}$ os autores afirmam que a recuperação das vítimas de TCE tem como fator determinante principal a gravidade do trauma e das lesões cranianas associadas, e a avaliação das sequelas tem demonstrado que os pacientes que tiveram danos encefálicos apresentaram pior prognóstico tanto em relação à mortalidade quanto à morbidade. No estudo de Dantas Filho et al., ${ }^{12}$ no qual se investigou a influência de vários fatores sobre a evolução de pacientes vítimas de TCE, o tipo de lesão intracraniana teve influência expressiva sobre a evolução desses pacientes.

Para Macedo ${ }^{3}$ e Carvalho et al., ${ }^{11}$ em estudo com crianças e adolescentes vítimas de TCE leve e grave, respectivamente, foi demonstrado que as lesões intracranianas (LIC) são as verdadeiras responsáveis pelo prognóstico dos pacientes com TCE. O estudo de Macedo $^{3}$ também mostra que a maior importância da ocorrência de sinais e sintomas nas crianças com TCE leve está em sua possível associação com fratura de crânio ou LIC, e estas últimas são as verdadeiras responsáveis pela morbidade e mortalidade resultantes desse tipo de trauma. Nesse estudo, o autor sugere que as crianças com fratura de crânio e tomografia computadorizada de crânio (TCC) inicial sem lesão intracraniana podem ser acompanhadas no domicílio, sem prejuízo ao prognóstico. Esse resultado corrobora os de outros autores que consideram que crianças e adolescentes com pontuação na ECG igual a 15, sem déficit neurológico focal e ausência de LIC na TCC inicial, não precisam ser internados, ou seja, sem detrimento quanto ao prognóstico.,11

No estudo de Dantas Filho et al.,12 a pressão arterial sistólica abaixo de $90 \mathrm{mmHg}$ e a associação de hipóxia e hipotensão arterial, quando presentes no momento da admissão, tiveram influência negativa sobre os pacientes, assim como no estudo de Carvalho et al., ${ }^{11}$ em que a hipóxia e a hipotensão também se associavam a esse fato.

Em estudo de Morgado e Rossi ${ }^{4}$ com pacientes vítimas de TCE atendidos nas primeiras 12 horas, observou-se que, com o aumento da idade, também houve aumento da gravidade do TCE, o que leva a pior prognóstico dessas vítimas. Esse estudo corrobora o de outros autores, tais como Muñoz-Céspedes et al. ${ }^{7}$ e Melo et al., ${ }^{1}$ que também afirmam que a maior idade (pacientes acima de 60 anos) é um fator relacionado com pior prognóstico em vítimas de TCE. Sousa ${ }^{8}$ também refere que no processo de recuperação após o TCE a idade exerce efeito deletério. Por outro lado, no estudo de Dantas Filho et al., ${ }^{12}$ a idade e os politraumatismos isoladamente não exerceram maior risco de agravamento no quadro clínico dos pacientes analisados. Nesse estudo, a gravidade foi desproporcionalmente menor entre os mais velhos, e esse fato não exerceu influência negativa da idade sobre o resultado. Esse autor afirma, ainda, que a desproporcionalidade entre as faixas etárias e a distribuição da gravidade têm como provável fator relacionado a grande incidência de traumas graves, incluindo o TCE, entre a população jovem mais exposta. ${ }^{12}$

\section{Considerações finais}

Nos últimos anos, têm-se aprofundado os estudos no intuito de se buscarem os fatores prognósticos para o TCE. A identificação desses indicadores tem representado um grande avanço na busca de alternativas para orientar o tratamento do paciente e estimar o resultado final.

Com base na literatura analisada, foi evidenciado que a gravidade inicial medida pela ECG tem sido o 
instrumento mais usado para avaliação do prognóstico funcional após o TCE, e uma menor pontuação nessa escala está relacionada a maior gravidade dos traumatismos cranioencefálicos e, consequentemente, é um dos principais fatores relacionados a pior prognóstico.

Ainda assim, a presença de lesões cerebrais, a gravidade das lesões, o tipo de lesão intracraniana, sequelas neurológicas, tempo de intervenções clínicas e cirúrgicas, comorbidades médicas, presença de febre, hipóxia, hipotensão arterial e a associação de hipóxia e hipotensão arterial também foram fatores determinantes sobre a evolução dos pacientes. Por outro lado, o politraumatismo não expressou significativamente a evolução desses pacientes. A faixa etária das vítimas e sua relação com pior prognóstico foram discordantes em alguns estudos, e a presença ou ausência de influência significativa da idade sobre a evolução dos pacientes vítimas de traumatismo craniano levanta uma importante e ampla discussão sobre esses casos.

Podemos ressaltar também que o TCE sem sido um dos principais motivos de procura por atendimento médico de urgência tanto de adultos quanto de crianças e adolescentes, podendo levar também a lesões de alta morbimortalidade.

Em relação aos acidentes que envolvem meios de transporte, é cada vez mais evidente a imprudência por parte dos motoristas, a má conservação das rodovias e também a falta de cuidado por parte dos pedestres, fatores esses que, somados, implicam uma maior prevalência no número de acidentes de trânsito e, consequentemente, a prevalência do alto índice de vítimas de TCE.

Assim, esforços maiores devem ser empreendidos para a prevenção e assistência em todos os níveis de atendimento, visando minimizar essa problemática, visto que um prognóstico confiável reflete em benefícios, além de orientação apropriada para o tratamento e a reabilitação do paciente.

\section{Referências}

1. Melo JRT, Oliveira Filho J, Silva RA, Moreira Júnior ED. Fatores preditivos do prognóstico em vítimas de trauma cranioencefálico. Arq Neuropsiquiatr. 2005;63(4):1054-7.
2. Moura JC, Rangel BLR, Creôncio SCE, Pernambuco JRB. Perfil clínico-epidemiológico de traumatismo cranioencefálico do Hospital de Urgências e Traumas no município de Petrolina, estado de Pernambuco. Arq Bras Neurocir. 2011;30(3):99-104.

3. Macedo KC. Características clínicas e epidemiológicas de crianças e adolescentes com traumatismo cranioencefálico leve e análise de fatores associados à fratura de crânio e lesão intracraniana [dissertação]. Belo Horizonte: Universidade Federal de Minas Gerais; 2006.

4. Morgado FL, Rossi LA. Correlação entre a escala de coma de Glasgow e os achados de imagem de tomografia computadorizada em pacientes vítimas de traumatismo cranioencefálico. Radiol Bras. 2011;44(1):35-41.

5. Andrade AF, Paiva WS, Amorim RLO, Figueiredo EG, Neto ER, Teixeira MJ. Mecanismos de lesão cerebral no traumatismo cranioencefálico. Rev Assoc Med Bras. 2009;55(1):75-81.

6. Pereira CU, Duarte GC, Santos EAS. Avaliação epidemiológica do traumatismo cranioencefálico no interior do estado de Sergipe. Arq Bras Neurocir. 2006;25(1):8-16.

7. Muñoz-Céspedes JM, Lapedriza NP, Valero CP, Ustarroz JT. Factores de pronóstico en los traumatismos craneoencefálicos. Rev Neurol. 2001;32(4)351-64.

8. Sousa RMC. Fatores de risco para dependência após trauma cranioencefálico. Acta Paul Enferm. 2005;18(4):35460.

9. Oliveira CO, Ikuta N, Regner A. Biomarcadores prognósticos no traumatismo cranioencefálico grave. Rev Bras Ter Intensiva. 2008;20(4):411-21.

10. Cambier J, Masson M, Dehen H. Neurologia. $11^{\text {a }}$ ed. Rio de Janeiro: Guanabara Koogan; 2005.

11. Carvalho LFA, Affonseca CA, Guerra D, Ferreira AR, Goulart EMA. Traumatismo cranioencefálico grave em crianças e adolescentes. Rev Bras Ter Intensiva. 2007;19(1):98-106.

12. Dantas Filho VP, Falcão ALB, Sardinha LAC, Facure JJ, Araújo S, Terzi RGG. Fatores que influenciaram a evolução de 206 pacientes com traumatismo cranioencefálico grave. Arq Neuropsiquiatr. 2004;62(2-A):313-8.

13. Jones Júnior HR. Neurologia de Netter. Porto Alegre: Artmed; 2006.

14. Alcalde TF, Regner A, Rodrigues Filho EM, Silveira PC, Grossi GG, Simon D. Ausência de associação entre polimorfismo do gene da interleucina-1 beta e o prognóstico de pacientes com traumatismo cranioencefálico grave. Rev Bras Ter Intensiva. 2009;21(4):343-8.

\section{Endereço para correspondência}

José Carlos de Moura

Rua Joaquim Nabuco, 798, Centro

56304-040 - Petrolina, PE, Brasil

E-mail: jcdemoura@uol.com.br 